\title{
VOLATILIDAD FISCAL Y CRECIMIENTO ECONÓMICO. VENEZUELA, 1998-2010
}

\author{
FINANCIAL VOLATILITY AND ECONOMIC GROWTH.
} VENEZUELA, 1998-2010

\author{
Carlos José Peña Parra \\ Universidad Central de Venezuela \\ Instituto de Investigaciones Económicas y Sociales \\ carlojosep@yahoo.com
}

\section{RESUMEN}

El objetivo del presente artículo es determinar el efecto de la volatilidad fiscal sobre el crecimiento económico para Venezuela entre 1998 y 2010. En este lapso, según los datos presentados, la volatilidad de los agregados fiscales ha sido sensiblemente mayor al promedio del crecimiento del producto. Diversos factores han ocasionado que dichos agregados hayan sido más volátiles; entre ellos, los diversos choques externos, la crisis global y, a nivel interno, su comportamiento procíclico, la discrecionalidad, la falta de reglas e instituciones inadecuadas, todos ellos pueden tener responsabilidad en el incremento de la volatilidad fiscal y, en consecuencia, sobre el crecimiento. La literatura especializada señala que el vínculo entre la volatilidad fiscal y el crecimiento económico es negativa.

Palabras clave: crecimiento, volatilidad fiscal, cointegración.

Clasificación JEL: E32, E62 


\begin{abstract}
The purpose of this paper is to determine the relationship between fiscal volatility and economic growth for Venezuela, between 1998 to 2010. During this period, the volatility of fiscal policy has been significantly above average output growth. Several factors have caused the fiscal policy has been more volatile, among them, the various external shocks, the global crisis and, internally, procyclical behavior, discretion, lack of rules and inadequate institutions, may have been responsible for the attorney increased volatility and consequently on growth. The literature indicates that the link between fiscal volatility and economic growth is negative
\end{abstract}

Keywords: growth, fiscal volatility, cointegration 


\section{INTRODUCCIÓN}

El impacto de los choques externos sobre las economías domésticas es particularmente relevante en países en desarrollo, en los cuales el sector público depende considerablemente de los ingresos aportados por las materias primas, como ocurre en algunas economías latinoamericanas. En estos casos, el papel de los términos de intercambio, en especial su volatilidad, tiene un efecto importante en los ingresos públicos y, en consecuencia, sobre la política fiscal. En particular los resultados fiscales tienden a ser volátiles.

Los factores externos no son los únicos causantes de volatilidad. Las políticas macroeconómicas domésticas pueden compartir la culpa, y no tanto por los errores en la conducción de la política fiscal, sino, sobre todo, por la ausencia generalizada de reglas e instituciones adecuadas que, sumadas a las deficiencias de los mercados financieros, restringen enormemente el margen de maniobra de la gestión macroeconómica. Los elementos antes planteados configuran un escenario para que la política fiscal sea muy volátil. La literatura señala que hay un vínculo negativo entre la volatilidad fiscal y el crecimiento económico.

En Venezuela, para el lapso 1998-2010, la volatilidad promedio de los agregados fiscales ha sido sensiblemente superior al promedio de crecimiento del producto; en particular, los ingresos fiscales son más volátiles que los otros agregados. Según la evidencia, para el lapso en estudio la volatilidad promedio de los ingresos fiscales fue cuatro veces mayor que la tasa de crecimiento económico medio, seguido de la volatilidad del resultado fiscal, la cual fue dos veces superior a la variación del producto. Las causas que han originado que la política fiscal haya sido en este período muy volátil pueden ser diversas, entre ellas los choques de origen 
externo, sin embargo, sumado a ellos están el comportamiento pro cíclico de la política fiscal, la discrecionalidad, la falta de reglas e instituciones inadecuadas. Dicha situación puede tener un impacto importante en el crecimiento económico. En este sentido, el objetivo del trabajo es determinar el efecto de la volatilidad fiscal sobre el crecimiento económico para Venezuela entre 1998 -2010. La metodología que se aplica para evaluar empíricamente el objetivo está basada en la cointegración y el modelo de corrección de errores. La estimación del vector de cointegración se llevó a cabo a través del método de los mínimos cuadrados completamente modificados, FMOLS y la estimación del modelo de corto plazo con corrección de errores se realizó con el método de los mínimos cuadrados ordinarios.

El trabajo está organizado de la siguiente manera: una sección introductoria, seguida de los elementos teóricos y empíricos que explican la relación entre volatilidad fiscal y crecimiento económico, posteriormente se abordan los principales hechos para la economía venezolana y la estimación de los modelos econométricos y se finaliza con las conclusiones.

\section{ALGUNOS ELEMENTOS TEÓRICOS Y EMPÍRICOS}

\subsection{Política fiscal y crecimiento económico}

Es posible que uno de los determinantes del bienestar económico y de los niveles de ingreso per cápita de una economía sea la política fiscal. El tamaño del sector público, las distintas funciones del gasto público y la estructura fiscal a través de la cual se financia la administración pública influyen en las decisiones económicas y en las actuaciones de los agentes económicos privados. Por tal razón, desde hace algún tiempo, los efectos 
de la política fiscal sobre el crecimiento económico a largo plazo han recibido una especial atención en la literatura sobre crecimiento económico, tanto a nivel teórico como empírico. En este contexto, en la última década han aparecido una gran cantidad de trabajos teóricos y empíricos con propuestas de cómo aumentar las tasas de crecimiento de la renta per cápita, modificando el nivel, la composición y financiamiento del gasto público. Diversas investigaciones han analizado la relación entre crecimiento económico y el nivel o composición del gasto público, entre ellas Kormendi y Meguire (1985), Barro (1989, 1990, 1991), Castles y Dowrick (1990), Doménech y Garcia (2001, 2002), Doménech (2004). La política fiscal teóricamente debería tener una función de estabilización automática; es decir, compensar los choques recesivos mediante la expansión de la demanda agregada y lo opuesto en el caso de los choques expansivos. Sin embargo, en América Latina es más frecuente el denominado comportamiento procíclico. Aquel que acentúa el comportamiento del ciclo económico en la bonanza y la recesión, respectivamente. Este desempeño refleja una incapacidad institucional y de funcionamiento de los mercados financieros a los cuales se tiene acceso. Asimismo, el desarrollo de la política fiscal en América Latina ha estado condicionado por factores de carácter externos, asociado a la evolución de los precios de las materias primas y los cambios en los flujos de capital de corto y largo plazo y, por factores internos, vinculados a la estructura económica de cada país y a los vaivenes políticos de cada uno de ellos. 


\subsection{Volatilidad de la política fiscal y crecimiento}

Uno de los elementos que afectan el crecimiento económico es la volatilidad de la política macroeconómica ${ }^{1}$; es decir, las políticas económicas como fuente de volatilidad endógena. Goyal y Sahay (2007) argumentan que la variabilidad de las políticas monetaria y fiscal; así como los abruptos cambios en las políticas de carácter estructural, pueden ser fuentes de volatilidad macroeconómica. Con base en este argumento, Fatas y Mihov (2005) encuentran una relación inversa entre la volatilidad de política económica y crecimiento. En particular, una política fiscal más volátil afecta negativamente a la inversión. Este vínculo puede ser más fuerte en países donde el ingreso per cápita sea bajo. Según los autores, una explicación de este hallazgo pueden ser las severas restricciones de financiamiento que enfrentan dichos países. Adicionalmente, aquellos países fiscalmente más volátiles serán también los de mayor incertidumbre macroeconómica y, por lo tanto, presentaran más dificultades para financiar su acumulación de capital.

Un conjunto de trabajos presenta evidencia a favor de la idea de que la volatilidad del gasto público afecta el crecimiento. Así, Alfonso y Furceri (2008) plantean que la volatilidad del gasto público puede tener efectos positivos o negativos sobre el crecimiento. Serían positivos cuando la volatilidad fuera utilizada como una forma de suavizar los choques que recibe la economía, afectando positivamente la inversión privada y el crecimiento de largo plazo. Por el contrario, tendría consecuencias negativas si esta volatilidad genera mayores fluctuaciones del ciclo económico y de la volatilidad

1 La evidencia teórica y empírica ha puesto de relieve la importancia de la volatilidad de las políticas macroeconómicas, ya que tiene efectos sobre los resultados macroeconómicos, en particular en América Latina. La interpretación de esto es quizás un poco difícil, ya que alguna variación en las políticas fiscales, monetarias o cambiarias generan impactos desestabilizadores sobre la economía; otras situaciones serían el resultado de elementos exógenos, como, por ejemplo, choques externos. 
macroeconómica, lo cual se daría en el caso de las políticas procíclicas. En este sentido, Roubini y Sachs (1989), Alt y Lowry (1994), Poterba (1994), Levinson (1998) y Lane (2002), muestran evidencia de los efectos de la volatilidad fiscal sobre el ciclo económico, observan que una menor volatilidad fiscal hace más lentos los ajustes de la economía a los choques negativos.

Fatas y Mihov $(2001,2003,2006)$ sostienen que, aunque la evidencia señala que tanto los efectos positivos y negativos sobre el crecimiento son significativos, predominan los negativos. En Fatas y Mihov (2003) se presenta evidencia empírica de la relación entre la volatilidad fiscal y el crecimiento y se concluye que si la volatilidad fiscal aumenta en una desviación estándar, el crecimiento económico disminuye 0.8 por ciento. Por otra parte, Goyal y Sahay (2007) con un enfoque basado en la identificación de los episodios de alto y bajo crecimiento para analizar las consecuencias del comportamiento de la política fiscal en cada uno de ellos, concluyeron que las variaciones del nivel de la volatilidad, según el tipo de episodio de crecimiento, son más importantes que el hecho de que la política fiscal sea procíclica.

Puede existir un conjunto de elementos que inciden en la volatilidad de la política fiscal. Los factores externos, tales como choques comerciales o financieros, tienen relevancia para la política macroeconómica, en particular la fiscal. Respecto a ello Catao (2007) sostiene que la influencia del ámbito externo constituye un elemento común en toda Latinoamérica. Goyal y Sahay (2007) han aportado otras pruebas y concluyen que en América Latina y el Caribe la volatilidad de los resultados y las políticas es mayor en los episodios de bajo crecimiento. Sin embargo, la inestabilidad que provocan las perturbaciones reales y financieras son similares en los niveles bajo y reducido de crecimiento. Por otro lado, Titelman, Pérez y Minzar (2008) sostienen que la dinámica del ciclo económico en América 
Latina y el Caribe ha obedecido históricamente a las variaciones de los términos de intercambio y a las corrientes financieras. La importancia relativa de estas ha variado en el tiempo. Córdova y Rojas (2010) plantean que para países exportadores de recursos primarios las altas volatilidades del gasto público y del crecimiento económico estarían asociadas a la volatilidad de los términos de intercambio. Por su parte, Collier y Goderis (2007) y Brahmbhatt y Canuto (2010) establecen que los efectos positivos o negativos de la dependencia de los productos primarios son condicionales al tipo de política fiscal que se adopte. Concluyen que aquellos países que tienen un gobierno débil y no poseen reglas, aumentarían su gasto público desmedidamente en los episodios de boom, lo que genera en el largo plazo una pérdida de bienestar y de crecimiento.

El efecto de los choques externos no sólo se refleja en el gasto sino también en los ingresos públicos. Kacef (2010) plantea que la volatilidad de los precios de exportación se traduce en una mayor volatilidad de los ingresos tributarios y de la política fiscal. Esto reduce el espacio fiscal y la capacidad de los gobiernos para enfrentar las demandas sociales. Situación que se magnifica en función del grado de apertura comercial o de internacionalización de una economía. Loayza y Raddatz (2007) sostienen que la apertura comercial puede magnificar los efectos de las perturbaciones en los términos de intercambio.

Otro elemento importante es que la volatilidad de los instrumentos fiscales empleados es un mejor indicador de la calidad de la política fiscal que las medidas basadas en los niveles de esos instrumentos (déficit fiscal). Adicionalmente a los efectos de los choques externos sobre la volatilidad de la política fiscal la literatura señala otro elemento de importancia, una política fiscal procíclica. El comportamiento pro cíclico refleja, en gran medida, la incapacidad de los gobiernos para financiar su gasto en tiempos 
difíciles a través de un aumento del ahorro público durante las épocas de auge. Un ejemplo de esta situación ha sido la gestión ineficiente de los ingresos fiscales extraordinarios derivados del aumento en los precios de las materias primas en países de América Latina, donde el sector público depende de dichos ingresos. Pero, el carácter procíclico de la política fiscal también refleja el funcionamiento imperfecto de los mercados de capitales. Una buena cantidad de estudios realizados en los últimos diez años, entre los cuales se pueden mencionar: Gavin y Perotti (1997), Kaminski, Reinhart y Végh (2004), Alesina y Tabellini (2005), Talvi y Végh (2005), han sostenido que la política fiscal de América Latina es procíclica; no obstante, es conveniente e importante destacar que, adicional a los efectos del nivel y prociclicidad de las políticas fiscales, es preciso considerar su volatilidad. Por su parte, Braun y Raddatz (2008) discuten extensamente que la prociclicidad de la política fiscal en las economías en desarrollo obedece a la debilidad de los instrumentos de política, y se ha atribuido el sesgo a la insolvencia de las cuentas fiscales a la interacción perversa entre la volatilidad externa y las características estructurales de la economía y los elementos de economía política. El resultado de estos factores influye para que la política fiscal sea volátil en América Latina.

En concreto, la política fiscal debería desempeñar un papel de estabilizador automático mediante un diseño adecuado de las estrategias de ingresos y gastos. En este sentido, Landerretche (2011) argumenta que hay consenso general sobre la importancia macroeconómica de evitar desequilibrios fiscales, especialmente en los países emergentes que suelen heredar problemas de credibilidad. Es decir, el gasto fiscal y las diferentes dificultades que se enfrentan cuando se trata de gobernar el proceso de presupuesto público generan un problema de gobernabilidad macroeconómica. Estas situaciones requerirían ajustes importantes a la institucionalidad macroeconómica. 
En síntesis, la breve revisión de la literatura pone de manifiesto la relación entre volatilidad fiscal y crecimiento económico y permite situar el contexto teórico y empírico de dicha relación.

\section{VENEZUELA: CRECIMIENTO Y VOLATILIDAD FISCAL}

\subsection{Choques externos, inestabilidad macroeconómica y política fiscal}

Como ya se mencionó, este trabajo se aborda para el periodo 1998 - 2010. Lapso que ha sido muy particular para el desempeño económico venezolano. Una etapa que estuvo signada por un conjunto de acontecimientos de orden político, económico y social. Una economía afectada por diversos choques externos, entre ellos: petroleros, negativos y positivos y crisis global. En lo interno, controles de precios, de tipo de cambio, devaluaciones, leyes regulatorias, expropiaciones y una política económica incoherente. Una política fiscal sujeta a restricciones financieras y, posteriormente, altamente expansiva.

Por ser la economía venezolana pequeña y abierta es extremadamente volátil. Sus causas pueden ser muchas; sin embargo, las principales están en los diversos choques externos, expresados a través de los términos de intercambio. Choques debidos, fundamentalmente, a la concentración de las exportaciones en muy pocos productos (petróleo). Su relevancia para explicar el comportamiento y dinámica de la economía venezolana no es un hecho sujeto a discusión.

En las últimas décadas la economía venezolana ha estado expuesta a constantes choques externos como consecuencia de la variabilidad de los precios petroleros, ${ }^{2}$ los cuales tienen un papel determinante en la volatili-

2 El sector petrolero incide positivamente en la economía interna a través de la gene- 
dad de los términos de intercambio. En particular, en el lapso 1998-2010 estuvieron presentes con más intensidad. A estos choques hay que agregar la crisis mundial del año 2008. En el caso venezolano, la trasmisión entre los choques externos, positivos y negativos y el resto de la economía pasan, necesariamente, por el espacio fiscal. Los choques externos alteran los ingresos y gastos fiscales y generan problemas de financiamiento del déficit fiscal. Con base en lo anterior, Jiménez y Tromben (2006) plantean que los resultados fiscales de los países especializados en productos no renovables, PNR, son en promedio más volátiles que la media de la región.

En las gráficas 1 y 2 se expone la evolución de los términos de intercambio y su volatilidad.

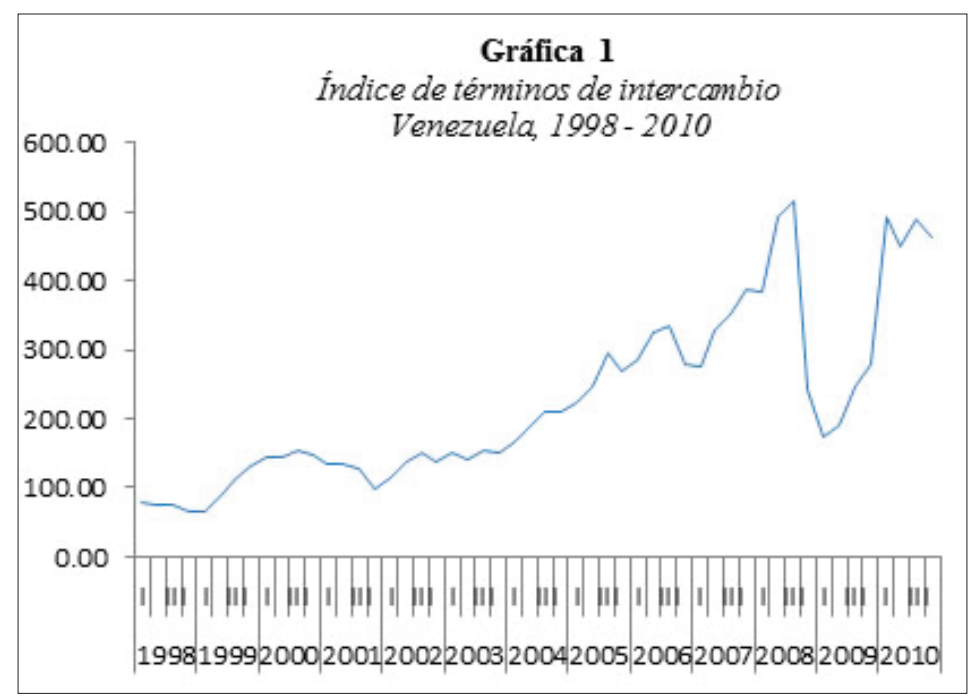

Fuente: Banco Central de Venezuela, BCV.

ración de divisas y de ingresos fiscales, los cuales constituyen fuentes de financiamiento de la demanda agregada doméstica; sin embargo, esta dependencia del sector petrolero hace que la actividad económica se encuentre sometida a un alto grado de volatilidad, en la medida en que se producen fluctuaciones importantes en los ingresos petroleros. 


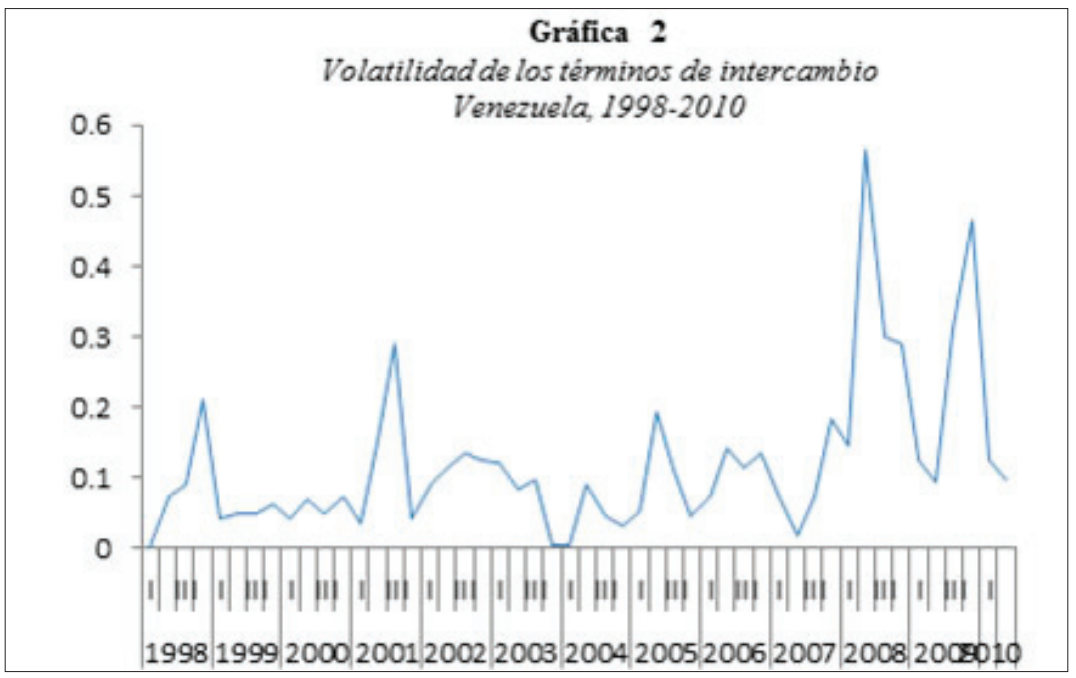

Fuente: Banco Central de Venezuela, BCV. Cálculos propios.

Como se muestra en las gráficas, el rasgo fundamental es la volatilidad, la cual viene dada en primer lugar por la fuerte variación en los precios del petróleo. Estas fluctuaciones tienen un efecto directo sobre el comportamiento y estructura del producto interno real, el tipo de cambio y los diferentes regímenes cambiarios. Los choques externos periódicos han determinado el carácter de los resultados fiscales, los cuales presentan una importante inestabilidad. Así, los choques externos y las oscilaciones de los resultados fiscales ${ }^{3}$ han tenido como elemento preponderante un comportamiento volátil y poco predecible del ingreso interno.

En tales condiciones las finanzas públicas venezolanas presentan las siguientes características: a) registran un déficit de magnitudes relevantes casi permanentes, b) niveles elevados de endeudamiento del sector público,

3 Otros elementos que podrían estar contribuyendo a la volatilidad de los agregados fiscales son el grado de profundidad del sistema financiero, rigideces en la estructura de ingresos y gastos; así como las rigideces político-institucionales. 
c) han introducido en la economía niveles de volatilidad crecientes. ${ }^{4}$ La volatilidad macroeconómica afecta la estabilidad, da lugar a cambios bruscos en la distribución del ingreso y en la asignación de los derechos de propiedad y, en consecuencia, se traduce en conflictos distributivos (CEPAL, 2008). Las características identificadas han condicionado el desempeño de la economía, en particular de la inversión privada y del crecimiento económico, y son el reflejo de una institucionalidad débil e inestable.

\subsection{Volatilidad de la política fiscal y crecimiento económico}

En general, la literatura especializada argumenta que la volatilidad de la política fiscal viene fundamentalmente por tres vías: impuestos, gasto y resultado fiscal. La volatilidad de estos ha sido y es una peculiaridad en América Latina. Situación que afecta la capacidad de los gobiernos para enfrentar sus compromisos, en especial, sus obligaciones de deuda. Las cifras de volatilidad de los agregados fiscales para Venezuela se muestran en el cuadro 1.

La división de los periodos obedece a los diversos acontecimientos que han afectado a la economía venezolana. Así, la primera etapa (19982001) estuvo signada por una caída en los precios del petróleo, restricciones fiscales y acontecimientos políticos de importancia: un nuevo gobierno, que ya lleva trece años, la aprobación de la Constitución Nacional en 1999, entre otros. En el periodo 2002-2003 continuaron las restricciones

4 Al ser el sector público un exportador neto grande, en relación con el resto de la economía, el ajuste del tipo de cambio nominal genera beneficios fiscales de considerables proporciones en el corto plazo. Por ello, el tipo de cambio se ha usado como mecanismo de corrección fiscal a pesar del costo en términos de inflación y nivel de actividad económica. En consecuencia, los impactos fiscales de los choques externos y el uso del tipo de cambio como mecanismo de corrección fiscal han creado una fuerte volatilidad en las variables macroeconómicas y un patrón de comportamiento procíclico en el gasto público. 
fiscales, en 2003 se establece un control de cambios que todavía prevalece. En el ámbito político se produce una huelga general, lo que ocasionó una caída sustancial del crecimiento económico y de los ingresos fiscales.

La etapa de 2004 a 2007 se caracterizó por el aumento sostenido de los precios del petróleo, lo que impulsó el gasto público y el uso de los fondos extrapresupuestarios. Tres premisas fundamentales sustentan la etapa: 1) El incremento en el gasto público ha sido superior a los ingresos fiscales; 2) aumento de la dependencia de los ingresos petroleros, en consecuencia la gestión fiscal se ha vuelto más vulnerable a la inestabilidad del mercado petrolero y 3) la falta de transparencia fiscal se ha hecho notable. Para el último lapso, 2008-2010, la situación cambia totalmente, se produce la crisis global, la cual afecta al mercado petrolero de forma negativa. Esta situación propició un sensible deterioro en las cuentas fiscales y en el crecimiento económico. En este sentido, Vera (2010) plantea que ninguna de las medidas tomadas encajó en lo que podría ser considerado un programa diseñado para atenuar el impacto recesivo de la crisis. En todo caso, las medidas tomadas lucían como reacciones más o menos automáticas de ajuste puramente fiscal. La reacción ante la crisis fue una política fiscal procíclica.

\section{Cuadro 1}

Volatilidad de los agregados fiscales. Venezuela, 1998-201

\begin{tabular}{|c|c|c|c|c|c|}
\hline & $1998-2001$ & $2002-2003$ & 2004-2007 & $2008-2010$ & $1998-2010$ \\
\hline$\sigma \operatorname{ImP}$ & 2,69 & 3,72 & 2,64 & 3,11 & 4,0 \\
\hline$\sigma c p u b$ & 1,19 & 1,40 & 0,92 & 1,18 & 1,2 \\
\hline$\sigma d e f / s u p$ & 1,71 & 3,25 & 3,36 & 2,11 & 2,6 \\
\hline бdef/sup* & 1,82 & 3,17 & 3,39 & 1.90 & 2,5 \\
\hline
\end{tabular}

Fuente: Ministerio del Poder Popular para las Finanzas Publicas. Cálculos propios

Nota: $\quad \sigma \mathrm{ImP}=$ volatilidad de los impuestos como $\%$ del PIB $\sigma c p u b=$ volatilidad del consumo público como $\%$ del PIB $\sigma$ def $/$ sup $=$ Volatilidad del déficit/superávit fiscal financiero como $\%$ del PIB $\sigma \mathrm{def} / \mathrm{sup}^{*}=$ volatilidad del déficit/superávit fiscal primario como $\%$ del PIB 
Es importante destacar que las cifras que se muestran en el cuadro son valores promedio por subperiodo. En este caso, se observa que la volatilidad promedio de los impuestos fue mayor a la del consumo público y a la del déficit/superávit fiscal global; a excepción de 20042007 , donde se presenta un aumento en la volatilidad promedio del déficit/superávit fiscal, debido fundamentalmente al choque petrolero positivo de aumento de los precios del petróleo de la cesta venezolana. Así mismo, se observan cambios importantes en la evolución de la volatilidad de estos agregados a lo largo de la muestra, producto de las diferentes situaciones descritas en los párrafos anteriores. Cualquiera que sea el agregado que se utilice, la volatilidad fiscal ha aumentado. En este sentido, Riutor y Zambrano (2002) consideran el período de 1970 a 1994 y hacen una comparación de Venezuela con la OECD y América Latina, para concluir que, para esa etapa, la volatilidad de los agregados fiscales en Venezuela fue superior a los otros dos. Así, la volatilidad de los ingresos fiscales supera cuatro veces la de la OECD y, según ellos, es bastante superior a la de los países de América Latina. Respecto al balance fiscal, la volatilidad de éste es del doble de los países de la OECD, aunque levemente inferior a algunos países de América Latina. Por su parte, Guzmán (2011) observa, en un estudio realizado para América Latina durante los años 1999 - 2008, que la volatilidad de los agregados fiscales de Venezuela es la más elevada de la región: la volatilidad de los ingresos fiscales se ubicó en promedio en 4.3 , la del déficit/superávit global en 6.1 y la del déficit/superávit primario en 6.3. Estas referencias sólo ratifican lo que se muestra en el cuadro 1. Los choques externos, la política económica discrecional, la falta de reglas fiscales, ${ }^{5}$ entre otros, pudieran explicar en buena me-

5 En Venezuela, en el lapso en estudio, la política fiscal ha estado condicionada, fuerte- 
dida esta evolución en sentido contrario a las tendencias de la región.

Riutor y Zambrano (2002), para el lapso 1970-1994, tratan de explicar cuánto de la volatilidad de los resultados fiscales se debe al entorno macroeconómico y cuánto a la discrecionalidad de la política fiscal en Venezuela. Con base en estimaciones econométricas concluyen que para ese período los factores determinantes del entorno macroeconómico explican una parte pequeña de la volatilidad de los resultados fiscales, medidos como déficit/superávit fiscales primarios/PIB. La mayor parte de la volatilidad se explica por la política fiscal discrecional. En cuanto a la posible relación entre la volatilidad de la política fiscal y el crecimiento económico se presenta el cuadro 2, donde se intenta establecer alguna relación.

\section{Cuadro 2}

Volatilidad de los agregados fiscales y crecimiento económico

Venezuela, 1998-2010

\begin{tabular}{|l|c|c|c|c|c|}
\hline & $1998-2001$ & $2002-2003$ & $2004-2007$ & $2008-2010$ & $1998-2010$ \\
\hline бImP & 2,69 & 3,72 & 2,64 & 3,11 & 4,0 \\
\hline ocpub & 1,19 & 1,40 & 0,92 & 1,18 & 1,2 \\
\hline odef/sup & 1,71 & 3,25 & 3,36 & 2,11 & 2,6 \\
\hline бdef/sup* & 1,82 & 3,17 & 3,39 & 1.90 & 2,5 \\
\hline Tpibpc & $-1,6$ & $-10,1$ & 9,6 & $-0,7$ & 1,2 \\
\hline Tpib & 0,4 & $-8,2$ & 12,1 & 0,3 & 2,6 \\
\hline
\end{tabular}

Fuente: Ministerio del Poder Popular para las Finanzas,

Banco Central de Venezuela, BCV y cálculos propios.

Notas: $\operatorname{sImP}=$ volatilidad de los impuestos como \% del PIB.

$\sigma \mathrm{cpub}=$ volatilidad del consumo público como $\%$ del PIB.

$\sigma$ def/sup $=$ Volatilidad del déficit/superávit fiscal financiero como \% del PIB.

Como se observa en el cuadro 2, entre 1998 y 2010 la volatilidad

mente, por una expansión importante del gasto, que genera un balance fiscal negativo. Al respecto, el difícil fiscal se ubicó en $2 \%$ en promedio anual. Otro elemento importante es el aumento del gasto, el cual promedió $25.2 \%$ como porcentaje del PIB; en tanto, que los ingresos como porcentaje del PIB se ubicaron, en promedio, en 23.2 por ciento. 
de los agregados fiscales fue superior a la tasa de crecimiento del Producto Interno Bruto per cápita, tPIBpc y a la del Producto Interno Bruto total, PIB. En particular, la volatilidad de los ingresos fiscales es tres veces superior a la tasa de crecimiento del tPIBpc. La elevada volatilidad ${ }^{6}$ de los ingresos fiscales obviamente repercute en la inestabilidad de los gastos fiscales, en especial, en los de inversión. Esta alta volatilidad de los ingresos también influye en el resto de la economía, lo que genera inestabilidad, inhibiendo la inversión y el crecimiento. Asimismo, la volatilidad del resultado fiscal, medido por el déficit/superávit fiscal global, resulta ser dos veces superior a la tasa de crecimiento del PIBpc. Quizás, lo importante a resaltar es que cualquiera que sea el agregado fiscal que se tome como referencia, la volatilidad de éste fue superior a la tasa de crecimiento, tanto del PIB, como del PIBpc. En todo caso, la volatilidad fiscal parece ser, según los resultados mostrados en el cuadro 2, una cuestión de mayor relevancia para la economía venezolana. Pareciera evidente que la inestabilidad fiscal tuvo consecuencias muy adversas, ceteris paribus, sobre el desempeño económico. Aunque los resultados deben ser interpretados con cautela, los mismos sugieren que la volatilidad de la política fiscal, efectivamente importa. Así, la política fiscal en Venezuela no es sólo volátil sino procíclica. El hecho de que posea esta característica se confirma no solamente en los episodios de choques externos adversos, sino también en los positivos (Riutor y Zambrano, 2002).

6 La excesiva volatilidad de los ingresos fiscales viene dada, entre otros aspectos, porque los ingresos fiscales presentan un alto componente exógeno al supeditarse a los ingresos provenientes de las exportaciones petroleras y, en consecuencia, tienden a moverse con los choques generados en el mercado petrolero. Pero también los ingresos fiscales no petroleros (impuesto sobre la renta no petrolera y los impuestos indirectos), a su vez, presentan una alta volatilidad, debido principalmente a la inestabilidad de las bases impositivas. 


\section{CONSIDERACIONES EMPÍRICAS PRELIMINARES}

\subsection{Datos y definición de variables}

El periodo muestral abarca desde 1998 a 2010 de manera trimestral. La fuente de los datos es el Banco Central de Venezuela, BCV, el Instituto Nacional de Estadísticas, INE, el Ministerio del Poder Popular para las Finanzas Públicas y cálculos propios.

Es importante definir y explicar el porqué de las variables a utilizar. En párrafos anteriores se definió el objetivo central del trabajo, el cual delimita su perfil. El manuscrito se centra en la volatilidad fiscal y sus consecuencias sobre el crecimiento económico. En este sentido, se utiliza como variable proxy de política fiscal el ratio consumo público/PIB, $c p u b$. La utilización de dicha variable está ampliamente documentada en la evidencia empírica. Por ejemplo, véase Cruz (2008), Ramírez (2006), entre otros. Una vez definido el agregado de política fiscal se procede a definir operacionalmente su volatilidad, misma que se mide a través de la desviación estándar del ratio consumo público/PIB. El uso de la desviación estándar para obtener la volatilidad de una variable en particular es un procedimiento convencional, reseñado en la literatura empírica sobre volatilidad. Al respecto, se puede revisar Ramey y Ramey (1995), Ramírez (2006), CEPAL (2008), entre otros, y para Venezuela, Riutort y Zambrano (2002). Malik (2002) incluye la desviación estándar del resultado fiscal como porcentaje del PIB, como medida de volatilidad fiscal, adicional a la volatilidad del consumo público/PIB.

Adicionalmente a las variables mencionadas en los párrafos anteriores, se incluye tasa de inversión privada, que se define como la formación bruta de capital fijo privado como porcentaje del PIB, tfbk. La 
inclusión de esta variable se sustenta en que, en el largo plazo, la inversión del sector privado constituye uno de los principales determinantes del crecimiento económico por su contribución al acervo de capital. En el corto plazo, las fluctuaciones de la inversión explican una proporción significativa de los cambios en el producto y en la demanda agregada.

\subsection{Prueba de raíces unitarias}

En cuanto a la estacionariedad, al desarrollar modelos de series de tiempo se necesita saber si el proceso estocástico que los generó es invariable en el tiempo. A este tipo de procesos se les denomina procesos estocásticos estacionarios. Si el proceso no es estacionario será muy difícil representar a la serie durante intervalos de tiempo pasados y futuros con un modelo algebraico simple. Si el proceso es estacionario, entonces es modelable mediante una ecuación de coeficientes fijos estimables con datos pasados. En la práctica es complicado encontrar series de tiempo surgidas de procesos estacionarios, sin embargo, hay técnicas que se encargan de convertir dichos procesos en estacionarios.

Las series no estacionarias son muy comunes en macroeconomía. Pueden ocurrir por diferentes razones y la razón subyacente suele tener importantes implicaciones para el tratamiento apropiado de la serie. Sería más sencillo si en términos relativos las series macroeconómicas sólo fueran estacionarias en tendencia, no obstante, una situación frecuente es aquella en que las series están sujetas a choques, bien sean coyunturales o estructurales, donde sus efectos no se extinguen con el tiempo.

Las pruebas de raíz unitaria se realizan para el logaritmo natural de las series en estudio, lpibpc, lfbk, locpub, lcpub, en nivel y para su prime- 
ra diferencia. En el primer caso la prueba incluye intercepto y tendencia determinista para analizar si la serie es estacionaria en torno a dicha tendencia. Para verificar si las variables presentan o no una raíz unitaria se recurre a las siguientes pruebas: Dickey-Fuller Ampliado o ADF (1981) y al de correcciones no paramétricas Phillips-Perron, PP, (1988).

\section{Cuadro 3}

Prueba de raíz unitaria

\begin{tabular}{|c|c|c|c|c|}
\cline { 2 - 5 } \multicolumn{1}{c|}{} & \multicolumn{2}{c|}{ DFA } & \multicolumn{2}{c|}{ PP } \\
\hline Variable & Estadístico $t$ & Critico $^{*}$ & Estadístico $t$ & Critico $^{*}$ \\
\hline lpibpc & $-1,7928$ & $-4,1809$ & $-4,0142$ & $-4,1484$ \\
\hline$\Delta$ lpibpc & $-6,9543$ & $-4,1809$ & $-22,4352$ & $-4,1525$ \\
\hline locpub & $-2,5832$ & $-4,1525$ & $-3,5004$ & $-4,1484$ \\
\hline$\Delta$ løcpub & $-7,0948$ & $-4,1525$ & $-16,4436$ & $-4,1525$ \\
\hline ltfbk & $-2,2313$ & $-4,1484$ & $-2,2313$ & $-4,1484$ \\
\hline$\Delta$ ltfbk & $-5,8235$ & $-4,1525$ & $-6,8235$ & $-4,1585$ \\
\hline lcpub & $-1,9897$ & $-4,1611$ & $-1,7508$ & $-4,1484$ \\
\hline$\Delta$ lcpub & $-5,6412$ & $-4,1611$ & $-20,3527$ & $-4,1525$ \\
\hline
\end{tabular}

Notas: *Nivel de significancia estadística: $1 \%$.

DFA: Dickey-Fuller Aumentado, PP: Phillips-Perron.

Hipótesis nula: ADF y PP la variable tiene raíz unitaria.

Como se observa en el cuadro 3, la evidencia señala que con la prueba ADF se acepta la hipótesis nula, igual con el PP; en este caso, las variables presentan una raíz unitaria, lo que implica que no son estacionarias. En consecuencia, con estas pruebas se puede decir que las series en niveles muestran una tendencia estocástica; no obstante, al diferenciarlas una vez, las variables son estacionarias con las pruebas anteriores. Por consiguiente, según la evidencia las variables son integradas de orden 1, es decir, son I(1). 


\section{MODELOS, ESTIMACIONES Y RESULTADOS}

\subsection{Consideraciones econométricas}

La evidencia empírica que se lleva a cabo tiene una doble función en la construcción y desarrollo de una teoría. En primer lugar, los datos validan o refutan las hipótesis asociadas a un modelo teórico específico. En segundo, los datos pueden sugerir la existencia de ciertas regularidades o "hechos estilizados" no contemplados inicialmente en la teoría y, de este modo, contribuir a orientar el esfuerzo de investigación.

Este ejercicio econométrico intenta realizar una evaluación de las relaciones estadísticamente significativas y estables de largo plazo (19982010) con base en una periodicidad trimestral. Así, dado que las variables en estudio resultaron ser integradas de orden I(1) se especifica y estima la siguiente función estática a largo plazo

$$
\text { lpibpc }=\alpha_{t}+\alpha_{1} l \sigma c p u b_{t}+\alpha_{2} l c p u b_{t}+\alpha_{3} l t f b k_{t}+{ }_{u} t
$$

Se espera que $\alpha_{1}<0 ; \alpha_{2} y \alpha_{3}>0$

En la ecuación (1), además de la volatilidad fiscal, locpub, se incluyen dos variables que tienen impactos positivos sobre el crecimiento económico, tanto a corto como a largo plazo: la política fiscal, lcpub y la tasa de inversión privada, ltfbkf.

Para establecer la dinámica de la volatilidad fiscal y su impacto en el crecimiento económico se especificó un modelo ad hoc que incorpora las relaciones de largo plazo, lo que se puede definir como su tendencia de largo plazo. Es decir, se desea precisar si las variables ${ }^{7}$ en estudio, lcpub, locupb,

7 Variables que posean una relación estacionaria en el tiempo con el crecimiento eco- 
ltfbkf, tienen efectos permanentes sobre la tendencia de largo plazo y sobre la dinámica de corto plazo de lpibpc, como variable proxy de crecimiento. Para obtener la relación de largo plazo se procedió de la siguiente manera: se utilizó el método propuesto por Phillips y Hansen $(1990)^{8}$ denominado mínimos cuadrados ordinarios, completamente modificados, FMOLS, para estimar el vector de cointegración, el cual presenta claras ventajas sobre los métodos de estimación más tradicionales disponibles en la literatura. Entre ellas, permite mayor robustez en las pruebas de hipótesis. Además, tal procedimiento lidia con los problemas de autocorrelación de las perturbaciones y la posible endogeneidad de los regresores. Las pruebas utilizadas para verificar la existencia de un vector de cointegración fueron las de Engle y Granger (1987) y Phillips y Ouliaris (1990), que están basados en los residuos. A continuación se presentan los resultados. Las salidas econométricas se encuentran en el anexo A, cuadros A1, A2 y A3.

\section{Cuadro 4}

Estimación de la ecuación de largo plazo

\begin{tabular}{|l|c|c|c|}
\hline \multicolumn{4}{|c|}{ Variable dependiente: lpibpc } \\
\hline \multicolumn{4}{|c|}{ Método: FMOLS } \\
\hline \multicolumn{4}{|c|}{ Periodo: 1998:1 - 2010:4 } \\
\hline Variable & Coeficiente & t-estadistico & Probabilidad \\
\hline C & 10,1627 & 25,4121 & 0,0000 \\
\hline locpub & $-0,0331$ & $-2,5146$ & 0,0155 \\
\hline Lcpub & 0,7831 & 7,0110 & 0,0000 \\
\hline Ltfbk & 0,2835 & 4,6393 & 0,0000 \\
\hline
\end{tabular}

nómico, en este caso con el PIB per cápita.

8 Es un método de estimación semiparamétrico que corrige el sesgo en muestras finitas del estimador mínimo cuadrado ordinario, MCO, ocasionado por la correlación serial de los errores de la cointegración y la endogeneidad en los regresores. El resultado es un estimador asintóticamente insesgado y completamente eficiente. Este método se utiliza para estimar el vector de cointegración en ecuaciones individuales. 


\begin{tabular}{|l|c|c|c|}
\hline $\mathrm{d} 03 \mathrm{q} 1$ & $-0,6112$ & $-5,4444$ & 0,0000 \\
\hline \multicolumn{3}{|c|}{ Estadísticos } & \\
\hline $\mathrm{R}^{2}$ & 0,606 & & \\
\hline Durbin-Watson & 1,982 & & \\
\hline
\end{tabular}

Fuente: Eviews.

\section{Cuadro 5}

Prueba de cointegración

\begin{tabular}{|l|c|c|}
\hline \multicolumn{1}{|c|}{ Prueba Engle- Granger } & Valor & Probabilidad \\
\hline Engle - Granger $t$ estadístico & $-6,090$ & 0,0005 \\
\hline Engle - Granger $z$ estadístico & $-43,383$ & 0,0004 \\
\hline \multicolumn{1}{|c|}{ Prueba Phillips - Ouliaris } & & \\
\hline Phillips - Ouliaris $t$ estadístico & $-6,187$ & 0,0004 \\
\hline Phillips - Ouliaris $z$ estadístico & $-47,957$ & 0,0001 \\
\hline
\end{tabular}

Nota: Hipótesis nula:las series no están cointegradas.

Fuente: Eviews.

El cuadro 4 muestra los resultados de la estimación del vector de cointegración por el método FMOLS. Se observa que las variables son estadísticamente significativas a $1 \%$ y $5 \%$ y con el signo correcto. Las variables se encuentran en logaritmos para interpretarlas en términos de elasticidades.

Para verificar si existe un vector de cointegración o una relación de largo plazo se observan los resultados mostrados en el cuadro 5, el cual despliega las pruebas de cointegración: Engle y Granger y Phillips y Ouliaris. Allí se verifica que, con ambos estadísticos, se rechaza la hipótesis nula de no cointegración a $1 \%$, lo que sugiere que las variables lcpub, locupb, ltfbkf y lpibpc, están cointegradas. En otras palabras, se sugiere que existe un proceso de convergencia dinámica y lineal en las variables indicadas, a lo largo de toda la muestra.

El análisis de cointegración se complementa con el estudio de la estabilidad de la relación de equilibrio, a través de una visualización gráfica 
de los contrastes de las pruebas de residuos recursivos y de la estimación recursiva de los coeficientes, con el objeto de identificar los choques exógenos en el periodo analizado. Esto, porque los choques tanto estructurales como coyunturales alteran el perfil de las variables, lo cual tiene consecuencias en el análisis de cointegración.

En cuanto a los efectos de la relación entre las variables del modelo, se consideró que los resultados de las pruebas de estabilidad pueden ser interpretados a través de la incorporación de una variable dummy a la regresión de cointegración, que resultó estadísticamente significativa. Una vez incluida la variable se obtiene un modelo razonablemente congruente, requisito inicial para un adecuado análisis del vector de cointegración.

Adicionalmente, se procedió a utilizar la prueba de Hansen (1992) para contrastar la consistencia de los parámetros del vector de cointegración, lo cual estaría indicando que el vector de cointegración es estable $y$, en consecuencia, la relación de largo plazo también. En este caso, la prueba utilizada por Hansen es el multiplicador de lagrange (Lc), conformándose así una prueba recursiva que se obtiene a partir de los residuos obtenidos de la relación de largo plazo, estimada por mínimos cuadros ordinarios completamente modificados para evitar el sesgo de simultaneidad que pudiera existir con regresores no exógenos. Es una prueba apropiada si se quiere verificar si existió un cambio de régimen en la relación bajo estudio. Los resultados se muestran en el cuadro 6.

\section{Cuadro 6}

Prueba de inestabilidad de Hansen

\begin{tabular}{|c|c|c|c|c|}
\hline Estadístico Lc & $\begin{array}{c}\text { Tendencias } \\
\text { estocásticas }(m)\end{array}$ & $\begin{array}{c}\text { Tendencias } \\
\text { determinanticas }(k)\end{array}$ & $\begin{array}{c}\text { Tendencias } \\
\text { excluidas }(p 2)\end{array}$ & Prob $^{*}$ \\
\hline 0,5855 & 4 & 1 & 0 & 0,0642 \\
\hline
\end{tabular}

Fuente: Eviews 7.2.

Notas: Hipótesis nula: Las series están cointegradas.

$\mathrm{Lc}(\mathrm{m} 2=2, \mathrm{k}=1) \mathrm{p}$-valores. Donde $\mathrm{m} 2=\mathrm{m}-\mathrm{p} 2$ es el número de tendencias estocásticas en una distribución asintótica. 
Según los resultados mostrados en el cuadro 6 se acepta la hipótesis nula, es decir, las variables en estudio están cointegradas, lo que valida la hipótesis de que el vector de cointegración es estable a lo largo de la muestra. Este resultado también parece indicar que la especificación correcta es lineal sin tendencia determinística.

Una vez realizadas las pruebas estadísticas pertinentes se procede a su interpretación económica. Las variables muestran el signo apropiado, según lo establecido en el modelo teórico, y son estadísticamente significativas. La evidencia mostrada en el cuadro 4 señala que la relación entre la volatilidad fiscal, locupb, y el crecimiento económico, lpibpc, presenta el signo correcto, es decir, aumentos en la volatilidad fiscal deterioran el crecimiento a largo plazo. No obstante, el impacto parece ser poco significativo, dado el valor del coeficiente de 0.03 . Por lo anterior, parece existir una relación inelástica entre ambas variables, ya que aumentos de $1 \%$ en la volatilidad fiscal disminuyen el crecimiento económico en 0.03 . Por su parte, la política fiscal, lcpub, y la tasa de inversión privada, $l t f b k$, generan un mayor crecimiento económico en el largo plazo. Así, la política fiscal, según la prueba, tiene una mayor significancia sobre el crecimiento a largo plazo. En cuanto a la variable dummy incorporada al vector, ella estaría afectando negativamente el crecimiento económico a largo plazo. Dicha variable representa parte de la huelga general llevada a cabo entre finales de 2002 y principios de 2003.

\subsection{Relación de corto plazo}

Una vez que se ha obtenido e interpretado la ecuación de largo plazo, el modelo dinámico de corto plazo se consigue, en este caso particular, a 
través de la incorporación de un modelo de corrección de errores con la estimación de un vector de cointegración, el cual se incorpora al modelo de corto plazo o dinámico mediante un modelo de corrección de errores, MCE, y la de largo plazo a través de los residuos del vector de cointegración. El modelo se especifica a continuación. Esto es válido ya que las series están integradas, lo que implica que existe una relación estable a largo plazo entre ellas, no obstante, en el corto plazo puede haber desequilibrio.

$$
\begin{aligned}
& \Delta l p i b p_{t}=\beta_{0}+\beta_{1} \Delta \sigma l c p u b_{t}+\beta_{2} \Delta l c p u b_{t}+\beta_{3} \Delta l t b k_{t}+\beta_{4} m c e_{t-}+\varepsilon_{t} \\
& m c e_{t}=l p i b p c_{t}-\beta_{0}-\beta_{1} \sigma c p u b_{t}-\beta_{2} l c p u b_{t}-\beta_{3} l t b k_{t}
\end{aligned}
$$

Las ecuación (2) describe la dinámica de corto plazo del PIB per cápita, lpibpc, en función de la volatilidad fiscal, la política fiscal y la tasa de inversión privada. La expresión (3) representa el modelo de corrección de errores, es decir, las desviaciones de la tasa de variación del PIB per cápita respecto de su nivel de equilibrio (determinado por las variables antes mencionadas). Los resultados se muestran en el cuadro 7. Las salidas econométricas se encuentran en el anexo B, cuadros B1-B5.

El término de error en la regresión de cointegración se interpreta como el error de equilibrio y es este, precisamente, el que sirve para relacionar la conducta de corto plazo de la variable con su valor a largo plazo.

El operador $\Delta$ indica la primera diferencia de las variables en logaritmos, lo que es equivalente a la tasa de crecimiento de las series; es el mecanismo de corrección de errores y es el parámetro de ajuste a corto plazo. La significancia estadística de dicho parámetro estaría indicando la proporción del desequilibrio en , que es corregido en el siguiente período. Mientras más cerca este de uno, más rápido será el ajuste hacia el equilibrio. 


\section{Cuadro 7}

Estimación de la dinámica de corto plazo

\begin{tabular}{|l|c|c|c|}
\hline \multicolumn{4}{|c|}{ Variable dependiente: $\Delta$ lpibpc } \\
\hline \multicolumn{4}{|c|}{ Periodo: $1998: 1-2010: 4$} \\
\hline Variable & Coeficiente & t-estadístico & Probabilidad \\
\hline C & 0,0015 & 0,2603 & 0,7960 \\
\hline$\Delta$ locpub & $-0,0218$ & $-4,9393$ & 0,0000 \\
\hline$\Delta$ lcpub & 0,5705 & 12,2431 & 0,0000 \\
\hline$\Delta$ ltfbk & 0,0981 & 2,2295 & 0,0315 \\
\hline MCE(-1) & $-0,3353$ & $-3,5794$ & 0,0009 \\
\hline D02q4 & $-0,1794$ & $-4,1174$ & 0,0000 \\
\hline D03q1 & $-0,4247$ & $-8,9281$ & 0,0000 \\
\hline D03q2 & 0,4100 & 8,9714 & 0,0000 \\
\hline D04q4 & 0,3106 & 7,6511 & 0,0000 \\
\hline D05q1 & $-0,3107$ & $-5,9652$ & 0,0000 \\
\hline \multicolumn{5}{|c|}{ Estadísticos básicos } \\
\hline R ${ }^{2}$ & 0,9030 & & 0,0000 \\
\hline F-estadístico & 41,4041 & & \\
\hline D.W & 2,3589 & & \\
\hline
\end{tabular}

Fuente: Eviews.

\section{Cuadro 8}

Prueba verificación de los supuestos del MCO

\begin{tabular}{|c|c|c|c|}
\hline \multicolumn{4}{|c|}{ Prueba de normalidad ${ }^{l}$} \\
\hline \multicolumn{4}{|c|}{ Test Jarque-Bera $1,1519(0,5621)$} \\
\hline \multicolumn{4}{|c|}{ Correlación Serial $^{2}$} \\
\hline \multicolumn{4}{|c|}{ Prueba LM (Breusch-Godfrey) } \\
\hline $\mathrm{F}$ - estadístico & 1,7111 & Prob F(2,38) & 0,1943 \\
\hline Obs* $R^{2}$ & 4,1309 & Prob Chi-cuadrado(2) & 0,1268 \\
\hline \multicolumn{4}{|c|}{ Prueba de homocedasticidad ${ }^{3}$} \\
\hline \multicolumn{4}{|c|}{ Prueba ARCH } \\
\hline $\mathrm{F}$ - estadístico & 1,4086 & Prob F $(1,47)$ & 0,2412 \\
\hline Obs* $\mathrm{R}^{2}$ & 1,4258 & Prob Chi-cuadrado(1) & 0,2324 \\
\hline
\end{tabular}




\begin{tabular}{|l|l|l|l|}
\hline \multicolumn{4}{|c|}{ Prueba Breusch-Pagan-Godfrey } \\
\hline F - estadístico & 0,7548 & Prob F $(9,40)$ & 0,6575 \\
\hline Obs* ${ }^{2}$ & 7,2587 & Prob Chi-cuadrado(9) & 0,6102 \\
\hline
\end{tabular}

Fuente: Eviews.

Nota: ${ }^{1}$ Ho: Los residuos se distribuyen normalmente.

${ }^{2}$ Ho: Los residuos no están correlacionados (no hay autocorrelación).

${ }^{3}$ Ho: Los residuos presentan varianza constante.

El modelo dinámico del PIB per cápita, lpibpc, se realizó mediante una estimación uniecuacional parsimoniosa con el método de los mínimos cuadrados ordinarios. Desde el punto de vista estadístico y económico el modelo es razonablemente aceptable. Los valores estadísticos presentados en el cuadro 8 indican que no existen los problemas que se analizan. Es decir, no hay violación de los supuestos del modelo clásico de mínimos cuadrados ordinario (MCO). Se incluyeron cinco variables dummy: 2002:4, $d 02 q 4,2003: 1, d 03 q 1,2003: 2, d 03 q 2 ; 2004: 4$, d04q4 y 2005:1, d05q1, las cuales corrigen choques coyunturales, dados los diversos choques, internos y externos, sufridos por la economía venezolana en el lapso en estudio y que afectan la dinámica del crecimiento económico en el corto plazo. Estas variables resultaron ser estadísticamente significativas y mejoran tanto el error de predicción como la robustez del modelo. Así mismo, de acuerdo con el estadístico $F$, la regresión resultó globalmente significativa con un margen de confianza de 99 por ciento.

El modelo estimado es acorde con lo planteado en la teoría. Las variables presentan los signos correctos y son estadísticamente significativos a 5\% y 1 por ciento. En consecuencia, según la evidencia, la variación del PIB per cápita o tasa de crecimiento económico, $\Delta l p i b p c$, está determinada por la variación de la volatilidad fiscal, $\Delta l \sigma c p u b$, la variación de la política fiscal, $\Delta l c p u b$ y la variación de la tasa de inversión privada, $\Delta l t f b k$. 
Al analizar los resultados obtenidos y mostrados en el cuadro 7 se puede notar que fluctuaciones en la tasa de volatilidad fiscal, $\Delta l \sigma c p u b$, generan cambios no persistentes en la tasa de inversión privada. Así, cada punto porcentual en el crecimiento de la volatilidad fiscal ocasiona una disminución de $0.02 \%$ en la tasa de crecimiento económico en el corto plazo. Por su parte, variaciones en la política fiscal, $\Delta l c p u b$, y en la tasa de inversión privada, $\Delta l t f b k$, tampoco producen cambios no persistentes en la tasa de crecimiento. De esta manera, cada punto porcentual en el crecimiento de estas variables resulta en un aumento de $0.57 \%$ y $0.09 \%$, respectivamente, en la tasa de crecimiento en el corto plazo.

De la interpretación de los resultados, tanto de largo como de corto plazo, se tiene que la volatilidad fiscal parece no tener un efecto significativo en el deterioro y disminución del crecimiento económico, ya sea a largo o corto plazo. Por su parte, la política fiscal genera un impacto mucho mayor sobre el crecimiento en el largo plazo y la importancia de la tasa de inversión privada sobre el crecimiento económico es más relevante en el largo plazo.

En cuanto al término de corrección de error, representado por la variable $\mathrm{MCE}$ en la regresión en diferencias del PIB per cápita, resultó con el signo esperado y estadísticamente significativo. El signo negativo del MCE indica que a mayor desequilibrio, mayores serán las presiones hacia su restablecimiento, es decir, el signo negativo asegura la estabilidad del equilibrio. Asimismo, representa la velocidad de ajuste del crecimiento económico al nivel de desequilibrio previo de largo plazo. Además, establece el vínculo entre el crecimiento, la volatilidad fiscal, la política fiscal y la tasa de inversión privada a largo plazo. En este caso, se puede decir que existe la presencia de un ajuste lento del crecimiento económico al equilibrio, que viene dado por el valor estimado del coeficiente de MCE sumamente bajo (0.335). Lo importante de tal resultado está en la interpretación que de él se hace. 
Para el caso analizado, que corresponde al periodo 1998-2010, han prevalecido los desequilibrios macroeconómicos, los choques externos negativos, entre ellos, la inestabilidad del mercado petrolero y crisis globales; así como también, crisis político-institucionales que han afectado sustancialmente la dinámica del crecimiento en Venezuela. En este sentido, el análisis lleva a los canales directos de la incidencia de variables mencionadas sobre el crecimiento y los efectos indirectos que se producen a través de los impactos de la política económica sobre el crecimiento económico.

\section{CONCLUSIONES}

El objetivo del trabajo estuvo centrado en determinar el efecto de la volatilidad fiscal sobre el crecimiento económico en Venezuela entre 1998 y 2010. Para ello se recurrió a la metodología econométrica, en particular el método de la cointegración y los modelos de corrección de errores.

Se ha constatado que los agregados fiscales en Venezuela muestran una excesiva volatilidad. La inestabilidad de los ingresos fiscales es muy superior a la de los otros agregados fiscales, incluso superior a los resultados fiscales. Factores como la inestabilidad de los precios del petróleo, expresados en la volatilidad de los términos de intercambio y la discrecionalidad de la política fiscal, son elementos que han influido en la volatilidad de los ingresos fiscales para el período en estudio. Así, la política fiscal en Venezuela, no únicamente es volátil sino también procíclica, hecho que se confirma no solamente en los episodios de choques externos adversos, sino de igual manera en los positivos. 
Los resultados de la aplicación de los métodos econométricos, cointegración y modelo de corrección de errores arrojaron que tanto en el largo como en el corto plazo el crecimiento económico en Venezuela está condicionado por la política fiscal, la volatilidad de la política fiscal, medida por la volatilidad del consumo público, como porcentaje del PIB, y la tasa de inversión privada. Según la evidencia, la relación entre la volatilidad fiscal y el crecimiento económico es negativa.

Así, de la interpretación de los resultados de largo y de corto plazo se puede extraer que la volatilidad fiscal no tiene un efecto significativo en el deterioro y disminución en el crecimiento económico, tanto en el largo plazo como en el corto. Por una parte, la política fiscal genera un impacto mucho mayor sobre el crecimiento en el largo plazo, y por otra, el efecto de la tasa de inversión privada sobre el crecimiento económico tiene un impacto mucho mayor en el largo plazo. Entre ambas variables, la que tiene un impacto mayor sobre el crecimiento económico en el largo plazo así como en el corto es la política fiscal.

La necesidad de contar con mecanismos amortiguadores, como fondos de estabilización, así como la obligación de reglas que lleven a ejecutar políticas de gasto, definidas en un horizonte de mediano y largo plazo, son necesidades de reforma, dado el contexto aquí expuesto.

\section{REFERENCIAS}

Alesina, A. y G. Tebellini. 2005. "Why is fiscal policy often procyclical? NBER Working Paper Series, núm. 11600.

Alfonso, A. y D. Furceri. 2008. "Government size, composition, volatility and economic growth", European Central Bank, Working Papers Series, núm. 849. 
Alt, J. y R. Lowry. 1994. "Divided government, fiscal institutions and budget deficits evidence for the states", American Political Science Review, 88: 811-828.

Arreaza, A., M.A. Fernández y D. Delgado. 2000. "La demanda de dinero en Venezuela, 1984-1999”, Banco Central de Venezuela, Serie Documentos de Trabajo, núm. 28

Barro, R. 1989. "A cross-country study of growth, saving and government”, NBER Working Paper Series, núm. 2855.

. 1990. "Government spending in a simple model of endogenous growth", Journal of Political Economy, 98(5): 103-126.

. 1991. "Economic growth in a cross section of countries", Quarterly Journal of Economics, 106 (2): 407-443.

Brahmbhatt, M. y O. Canuto. 2010. "Natural resource and development strategy after de crisis", Economy Premise, núm. 1, Banco Mundial.

Braun, M. y Raddatz, C (2008) "The politics of financial development: evidence form trade liberalization", Journal of Finance, 63(3): 1469-1508

Castles, F. y S. Dowrick. 1990. "The impact of government spending levels on medium-term economic growth in the OECD, 1960-85", Journal of Theoretical Politics, 2: 173-204.

Collier, P. y B. Goderis. 2007. "Commodity prices, growth and the natural resource curse: reconciling and conundrum", Centre for the Study of African Economies, CSAE WPS/2007-15.

Córdova, J.P. y Y. Rojas. 2010. "Reglas fiscales y términos de intercambio", Revista Estudios Económicos, 19: 7-32.

Catao, L. 2007. "Retrospectivalatinoamericana", Finanzas \& Desarrollo. diciembre: $39-43$.

Comisión Económica para América Latina y el Caribe (CEPAL). 2008. Estudio económico para América Latina y el Caribe 2007-2008, Santiago de Chile. 
Cruz, P. 2008. Determinantes de la volatilidad del crecimiento económico en la historia económica de Chile: 1870-200, tesis de maestría en Economía, Pontificia Universidad Católica de Chile.

Dickey, D.A. y W.A. Fuller. 1981. "Likelihood ratio statistic for autoregressive time series with a unit root", Econometrica, 49: 1057-1072 Doménech, R. y J.R. García. 2001. "Estructura fiscal y crecimiento económico en la OCDE” Investigaciones Económicas, 25(3): 441-472. .2002. "Optimal taxation and public expenditure in a model of endogenous growth", Topics in Macroeconomics, 2(1).

Doménech, R. 2004. "Política fiscal y crecimiento económico", Universidad de Valencia (mimeo).

Engle, R.F. y C.W.J. Granger. 1987. "Co-integration an error correction: representation, estimation and testing" Econometrica, 55(2): 251-276.

Fatas, A. e I. Mihov. 2001. "The effects of fiscal policy on consumption and employment: theory and evidence", $\underline{\text { CEPR Discussion Papers }}$ núm., 2760.

. 2003. "The case for restricting fiscal policy discretion", The Quarterly Journal of Economics, 118(4): 1419-1447.

. 2005. "Policy volatility, institutions and economic growth", $\underline{\text { CEPR }}$ Discussion Papers, núm. 5388.

(2006) "The macroeconomic effects of fiscal rules in the US states", Journal of Public Economics, 90(1-2): 101-117.

Jiménez, J. P y V. Tromben. 2006. "Política fiscal en países especializados en productos no renovables en América Latina”, Serie Macroeconomía del Desarrollo, núm. 46, CEPAL.

Gavin, M y Perotti, R (1997) "Fiscal policy in Latin America" NBER Macroeconomics Annual. Vol. 12: 11-72 
González, M., Beyaert, A y Garcia, S (1999) "Integración y convergencia de los tipo de interés" Información Comercial Española, ICE. Revista de Economía. N $\mathrm{N}^{\circ}$ 782: 89-97

Goyal, R. y R. Sahay. 2007. "Volatility and growth in Latin American: an episodic approach”, FMI, documento de trabajo, núm. 06/287.

Granger, C.W.J. 1969. "Investigating causal relations by econometric models and cross - spectral methods", Econometrica, 37(3): 424-438.

Gregory, A.W. y B.E. Hansen. 1996. "Residual-based test for cointegration in models with regimen shifts", Journal of Econometrics, 70: 99-126.

Guzmán, R. 2011. "Volatilidad macroeconómica y política fiscal en El Salvador”, J.M. Fanelli, J.P. Jiménez y O. Kacef (comps.) Volatilidad macroeconómica y respuesta de políticas, Naciones Unidas.

Hansen, B. 1992. "Test for parameter instability in regressions with I(1) processes", Journal of Business and Economic Statistics, 10(3): 321-335.

Kacef, O. 2010. "Volatilidad macro-fiscal y gobernabilidad democrática", , CEPAL, Santiago de Chile.

Kormendi, R.C y Maguire, P.G (1985) "Macroeconomic determinants of growth: cross-country evidence" Journal of Monetary Economic. Vol 16(2): 141-164

Kaminski, G., C. Reinhart y C. Vegh. 2004. "When it rain, it pours: procyclical capital flows macroeconomics policies", NBER Working Papers Series, Nº 10780

Lane, Ph. 2002. "The cyclical behavior of fiscal policy: evidence from the OECD”, Trinity Economics Papers, núm. 20022.

Landerretche, O. 2011. "Gobernabilidad democrática, desarrollo institucional y crecimiento económico: una revisión de la literatura a la luz de la experiencia latinoamericana", Universidad de Chile, Serie documentos de trabajo, núm. 334. 
Levine, R. 2007. "Finance and growth: theory and evidence", en P. Aghion y S. Durlauf (comps.) Handbook of Economic Growth, 1a edición, volume 1, capítulo 12, Elsevier, pp. 865-934.

Levinson, A. 1998 "Balance budgets and business cycles: evidence from the States", National Tax Journal, 51(4): 715-732.

Loayza, N. y C. Raddatz. 2007 "The structural determinants of external vulnerability", The World Bank Economic Review, 21(3): 359-387.

Malik, A. 2002. "Volatilidad del producto en América Latina: resistencia de las economías y el papel de las instituciones", documento de trabajo, Proyecto andino de competitividad, CAF.

Poterba, J. 1994. "State response to fiscal crises: The effects of budgetary institutions", Journal of Political Economy, 102(4): 799-821

Phillips, P.C.B y B.E. Hansen. 1990 "Statistical inference in instrumental variables regressions with I (1) processes", Review of Economics Studies, 57(1): 99-125.

Phillips, P.C.B y S. Ouliaris. 1990. “Asymptotic properties of residual based test for cointegration", Econometrica, 58(1): 165-193.

Phillips, P y P. Perron. 1988. "Testing for a unit root in time series regression”, Biometrika, 75(2): 335-346

Ramey, G y V. Ramey. 1995. "Cross country evidence on the link between volatility and growth”, American Economic Review, 85(5): 1138-1151.

Ramírez R, N. (2006) "Efectos no lineales de la volatilidad sobre el crecimiento en economías emergentes", Banco Central de Reserva del Perú. Documento de Trabajo, DT. N²006-008.

Riutort, M. y L. Zambrano. 2002. "Volatilidad de la política fiscal en Venezuela”, Revista Cuadernos de Economía. Vol. 25(69): 111-115. Universidad Autónoma de Madrid 
Roubini, N y J. Sachs. 1989. "Government spending and budget deficits in the industrialized countries", Economy Policy, 8: 99-132.

Sims, C. 1972. "Money, income and causality", American Economic Review, 62(4): 540-552.

Talvi, E y Végh, C. 2005. "Tax base variability and procyclical fiscal in developing countries", Journal of Development Economics, 78(1): 156-190.

Titelman, D., E. Perez y R. Minzar. 2008. "Comparación de la dinámica de los efectos de los choques financieros y los choques de términos de intercambio en América Latina en el período 19802006", Serie de Financiamiento para el Desarrollo 203. CEPAL, Chile

Toledo, M. 2008. "Understanding business cycles in Latin America", documento presentado en el taller: Política macroeconómica y fluctuaciones cíclicas, CEPAL, mimeo

Vera, L. 2010. "La economía venezolana tras la crisis económica global", Boletín Económico Mensual, marzo, Banco Mercantil, Caracas, Venezuela. 


\section{ANEXO A}

\section{Cuadro A1}

\section{Estimación del vector de cointegración}

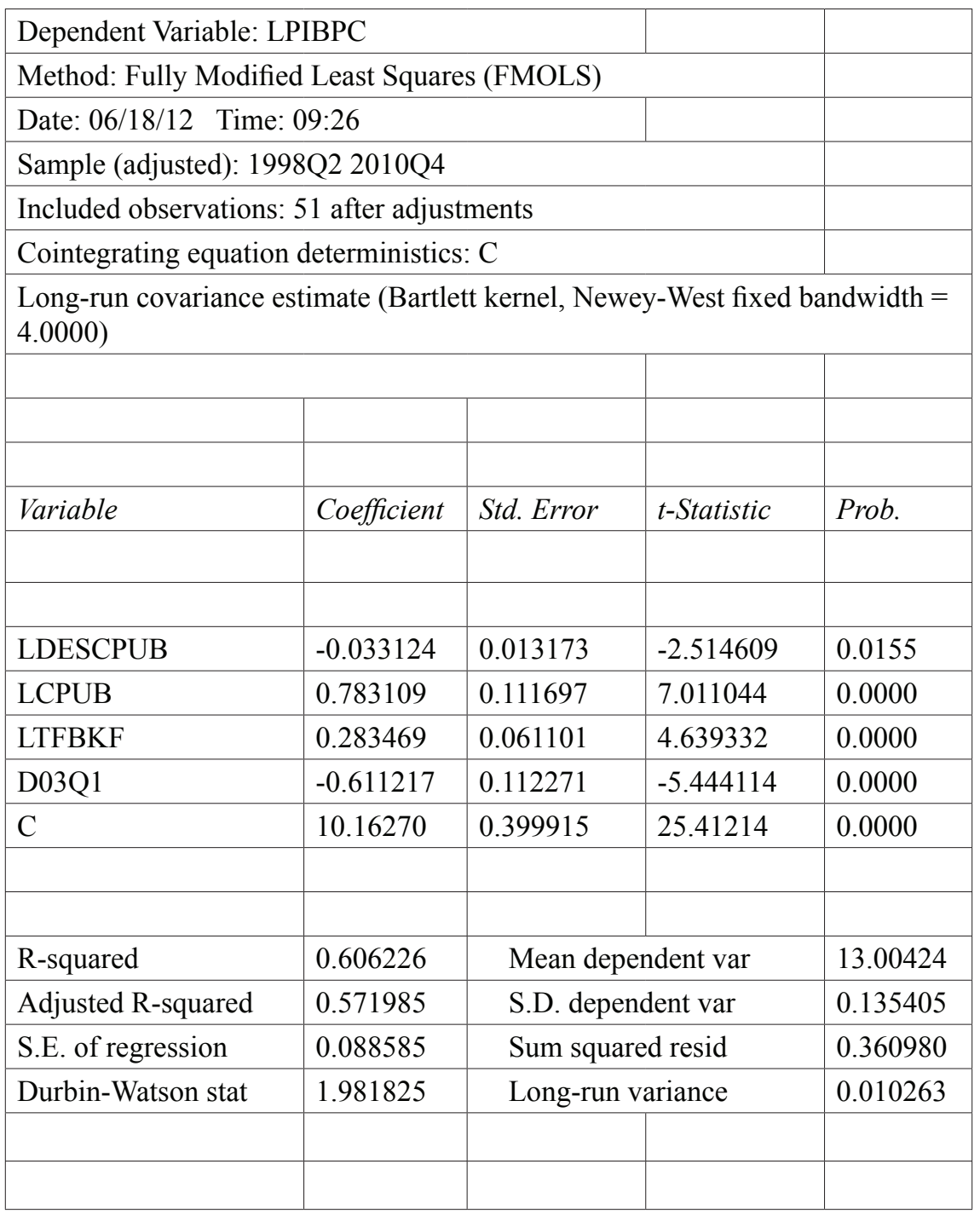




\section{Cuadro A2}

Prueba de Engle-Granger

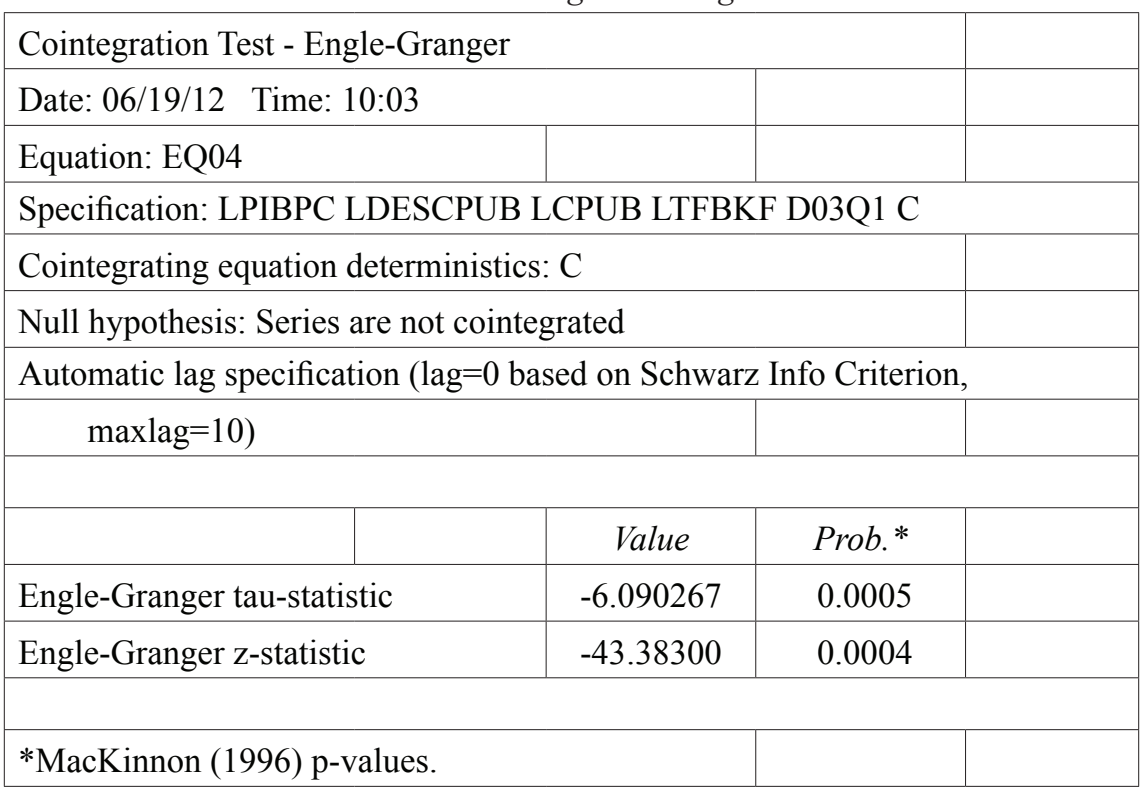

\section{Cuadro A3}

Prueba de Phillips-Ouliaris

\begin{tabular}{|c|c|c|c|}
\hline \multicolumn{3}{|c|}{ Cointegration Test - Phillips-Ouliaris } & \\
\hline \multicolumn{2}{|l|}{ Date: 06/19/12 Time: 10:05 } & & \\
\hline \multicolumn{2}{|l|}{ Equation: EQ04 } & & \\
\hline \multicolumn{4}{|c|}{ Specification: LPIBPC LDESCPUB LCPUB LTFBKF D03Q1 C } \\
\hline \multicolumn{3}{|c|}{ Cointegrating equation deterministics: $\mathrm{C}$} & \\
\hline \multicolumn{3}{|c|}{ Null hypothesis: Series are not cointegrated } & \\
\hline \multicolumn{4}{|c|}{$\begin{array}{l}\text { Long-run variance estimate (Bartlett kernel, Newey-West fixed bandwidth = } \\
4.0000)\end{array}$} \\
\hline & Value & Prob. * & \\
\hline Phillips-Ouliaris tau-statistic & -6.187481 & 0.0004 & \\
\hline Phillips-Ouliaris z-statistic & -47.95755 & 0.0001 & \\
\hline
\end{tabular}




\section{ANEXO B}

\section{Cuadro B1}

Estimación dinámica o de corto plazo

\begin{tabular}{|c|c|c|c|c|}
\hline \multicolumn{3}{|c|}{ Dependent Variable: D(LPIBPC) } & & \\
\hline \multicolumn{3}{|c|}{ Method: Least Squares } & & \\
\hline \multicolumn{3}{|c|}{ Date: $06 / 18 / 12$ Time: 09:42 } & & \\
\hline \multicolumn{4}{|c|}{ Sample (adjusted): 1998Q3 2010Q4 } & \\
\hline \multicolumn{4}{|c|}{ Included observations: 50 after adjustments } & \\
\hline Variable & Coefficient & Std. Error & t-Statistic & Prob. \\
\hline $\mathrm{C}$ & 0.001575 & 0.006050 & 0.260255 & 0.7960 \\
\hline D(LDESCPUB) & -0.021816 & 0.004417 & -4.939369 & 0.0000 \\
\hline $\mathrm{D}(\mathrm{LTFBKF})$ & 0.098145 & 0.044021 & 2.229534 & 0.0315 \\
\hline $\mathrm{D}(\mathrm{LCPUB})$ & 0.570508 & 0.046598 & 12.24310 & 0.0000 \\
\hline RESID05(-1) & -0.335372 & 0.093695 & -3.579387 & 0.0009 \\
\hline D03Q1 & -0.424715 & 0.047571 & -8.928062 & 0.0000 \\
\hline D03Q2 & 0.410030 & 0.045704 & 8.971434 & 0.0000 \\
\hline D04Q4 & 0.318791 & 0.041666 & 7.651155 & 0.0000 \\
\hline D05Q1 & -0.310696 & 0.052084 & -5.965291 & 0.0000 \\
\hline D02Q4 & -0.179468 & 0.043587 & -4.117422 & 0.0002 \\
\hline R-squared & 0.903063 & \multicolumn{2}{|c|}{ Mean dependent var } & 0.002676 \\
\hline Adjusted R-squared & 0.881252 & \multicolumn{2}{|c|}{ S.D. dependent var } & 0.116336 \\
\hline S.E. of regression & 0.040089 & \multicolumn{2}{|c|}{ Akaike info criterion } & -3.418558 \\
\hline Sum squared resid & 0.064286 & \multicolumn{2}{|c|}{ Schwarz criterion } & -3.036154 \\
\hline Log likelihood & 95.46395 & \multicolumn{2}{|c|}{ Hannan-Quinn criter. } & -3.272936 \\
\hline F-statistic & 41.40413 & \multicolumn{2}{|c|}{ Durbin-Watson stat } & 2.358906 \\
\hline Prob(F-statistic) & 0.000000 & & & \\
\hline
\end{tabular}




\section{Cuadro B2}

Prueba de normalidad de los residuos

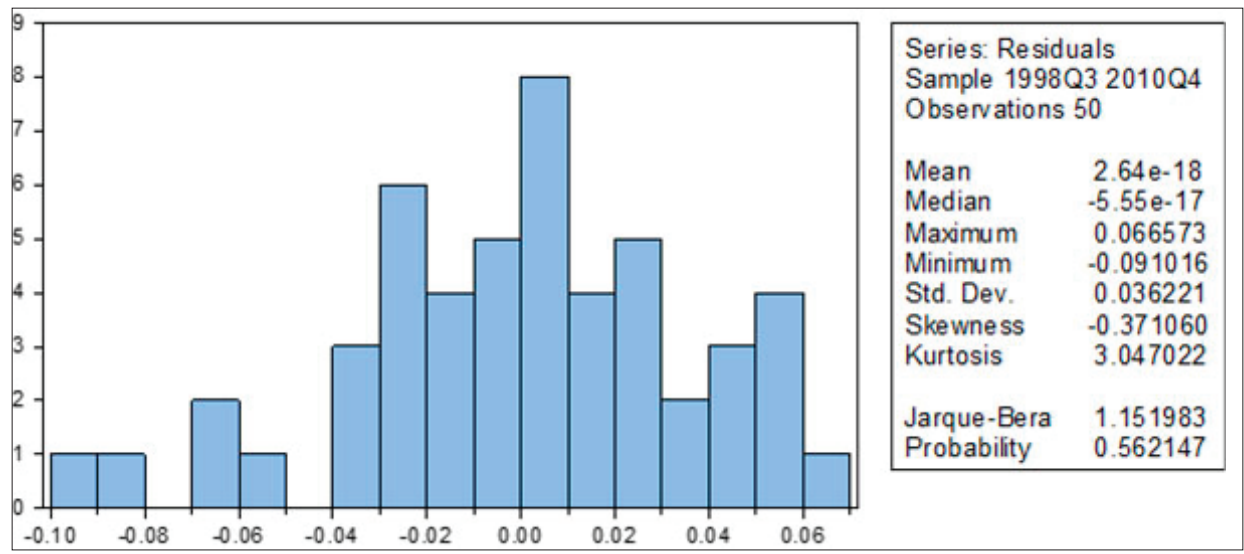

\section{Cuadro B3}

Prueba de correlación serial de los residuos

\begin{tabular}{|l|r|l|r|}
\hline \multicolumn{3}{|l|}{ Breusch-Godfrey Serial Correlation LM Test: } \\
\hline F-statistic & 1.711139 & Prob. F(2,38) & 0.1943 \\
\hline Obs*R-squared & 4.130963 & Prob. Chi-Square(2) & 0.1268 \\
\hline
\end{tabular}

\section{Cuadro B4}

\begin{tabular}{|c|c|c|c|c|}
\hline \multicolumn{4}{|c|}{ Heteroskedasticity Test: ARCH } & \\
\hline F-statistic & 1.408651 & Prob. F(1,47) & 0.2412 \\
\hline Obs*R-squared & 1.425858 & Prob. Chi-Square(1) & 0.2324 \\
\hline
\end{tabular}

\section{Cuadro B5}

Prueba de homocedasticidad

\begin{tabular}{|l|r|c|r|}
\hline Heteroskedasticity Test: Breusch-Pagan-Godfrey \\
\hline F-statistic & 0.754804 & Prob. F(9,40) & 0.6575 \\
\hline Obs*R-squared & 7.258782 & Prob. Chi-Square(9) & 0.6102 \\
\hline Scaled explained SS & 4.754844 & Prob. Chi-Square(9) & 0.8551 \\
\hline
\end{tabular}

